\title{
In Situ Correlative Imaging and Spectroscopy of Boehmite Particles in Liquid
}

\author{
Xiao-Ying $\mathrm{Yu}^{1}$, Juan $\mathrm{Yao}^{1}$, Bruce $\mathrm{Arey}^{2}$, Zihua $\mathrm{Zhu}^{3}$, and Jaehun Chun ${ }^{2}$ \\ 1. Earth and Biological Sciences Directorate, Pacific Northwest National Laboratory (PNNL), Richland, \\ WA, USA. \\ 2. Energy and Environment Directorate, PNNL, WA, USA. \\ 3. W. R \& Wiley Environmental Molecular Science Laboratory, PNNL, Richland, WA, USA.
}

This work presents an example of in situ imaging of boehmite $(\mathrm{AlOOH})$ particles, suspended in liquid, in a vacuum compatible microfluidic sample holder using a suite of tools including scanning electron microscopy (SEM) and time-of-flight secondary ion mass spectrometry (ToF-SIMS), highlighting the advantage of multiscale analysis in material sciences. Nanometer-sized boehmite particles exist in highlevel radioactive wastes at the Hanford site. [1] It is known that they are difficult to dissolve and cause rheological problems for processing in the nuclear waste treatment plant. Therefore, it is important to understand how boehmite particles form aggregates in waste tanks. Of particular interest is the $\mathrm{pH}$ effect on the boehmite aggregation and morphological change simulating tank waste relevant conditions.

A transferrable and vacuum compatible microfluidic interface, System for Analysis at the Liquid Vacuum Interface (SALVI), was used in this study. SALVI enabled surface analysis of liquids and liquid-solid interactions using ToF-SIMS and SEM $[2,3]$. Its detection windows consist of multiple apertures of $2 \mu \mathrm{m}$ in diameter open to vacuum, permitting direct probing of the liquid surface. Liquid is withheld by surface tension within the aperture. The vacuum compatible interface is composed of a silicon nitride membrane and polydimethylsiloxane microchannel $[2,3]$. A variety of samples including complex liquid mixtures, ionic liquids, single mammalian cells, live biofilms, and solid-electrolyte interface (SEI) have been studied using in situ imaging $[4,5]$. The feasibility of using SALVI for in situ characterization of nanoparticles in liquid was demonstrated in our previous work [6, 7]. This paper shows most recent results of correlative imaging of synthesized polydisperse boehmite particles under various $\mathrm{pH}$ conditions in deionized (DI) water.

High vacuum conditions were employed in liquid SEM (FEI Quanta). Figure 1 depicts SEM secondary electron (SE) imaging results of boehmite particles under wide $\mathrm{pH}$ conditions in the microchannel. The SE images show the synthesized particle size ranges from $30 \mathrm{~nm}-100 \mathrm{~nm}$. In situ liquid SEM provides descriptions of particle size, shape, morphology enhanced with elemental mapping. When comparing particle morphology and shape changes under different $\mathrm{pH}$ conditions, more aggregates are seen. It appears that the particles at high $\mathrm{pH}$ are not as dense as that at low $\mathrm{pH}$, this may be due to dissolution of boehmite under alkaline conditions. In situ liquid SIMS is used to study particle molecular structure and composition. Figure 2 depicts a comparison of positive ion mass spectra acquired using in situ liquid ToF-SIMS (IONTOF GmbH, TOF-SIMS V) under acidic, natural and alkaline conditions. The latter is used to simulate caustic conditions found in the nuclear tank wastes. The solubility of boehmite depends on $\mathrm{pH}$ [1]. The mass spectra provide direct evidence of this phenomenon. Both water clusters labelled in red marks and representative peaks vary as a result of $\mathrm{pH}$ change. SIMS provides detailed submicron molecular mapping of the particle and its surrounding water cluster environments as well as molecular identification of small molecules in liquid. These new results demonstrate advancements of in situ correlative imaging of liquid surfaces and solid-liquid interfaces using a universal microfluidic interface, SALVI $[2,3,8]$. 
References:

[1] S Clark et al., Basic Research Needs for Environmental Management, (2016), Department of

Energy. https://science.energy.gov/ /media/bes/pdf/reports/2016/BRNEM_rpt.pdf.

[2] L Yang et al., J. Vac. Sci. Technol. A 29 (2011), art. no., 061101. doi: 10.1116/1.3654147.

[3] L Yang et al., Lab Chip 11 (2011), p. 2481. doi: 10.1039/c01c00676a.

[4] H Xin et al., Int. Bio. 8 (2016), p. 635. doi: 10.1039/c5ib00308c.

[5] J Yu et al., Chem. Comm. 52 (2016), p. 10952. doi: 10.1039/c6cc02893d.

[6] L Yang et al., Surface Interface Analysis 46 (2015), p. 224. doi: 10.1002/sia.5252.

[7] J Yao et al., J. Vis. Exp 127 (2017), p. e56058. doi: 10.3791/56058.

[8] We acknowledge support from the PNNL Nuclear Science Processing Initiative (NPSI) Laboratory Directed Research and Development fund and instrument access to the DOE BER EMSL user facility under the General User Proposal 50143.
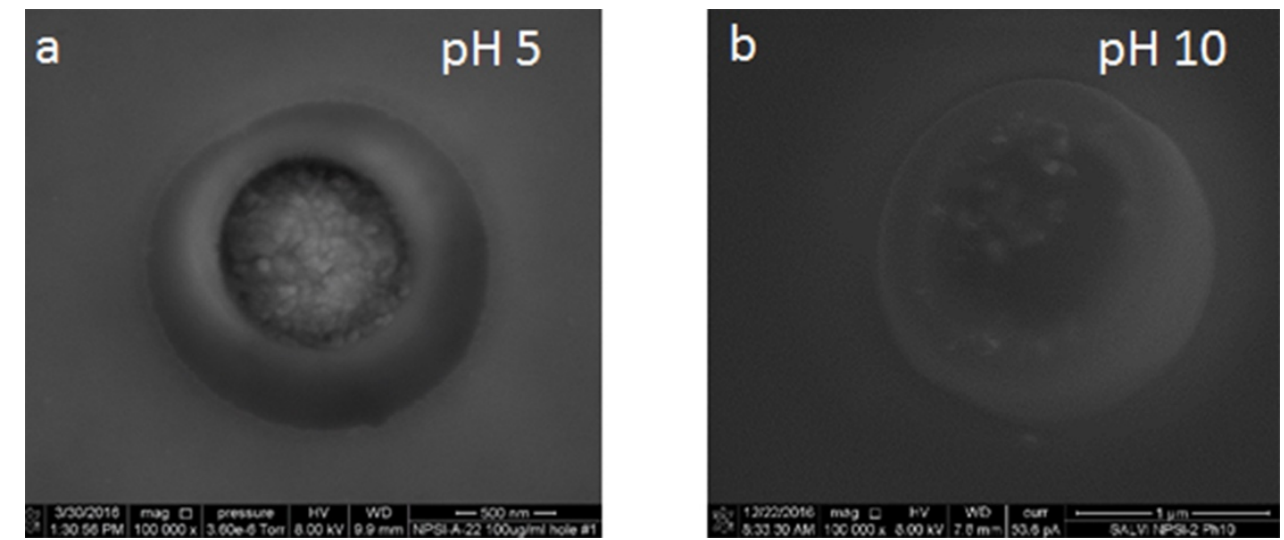

Figure 1. Liquid SEM SE imaging of boehmite particles in different $\mathrm{pH}$ conditions: (a) $\mathrm{pH} 5$ and (b) $\mathrm{pH}$ 10 .
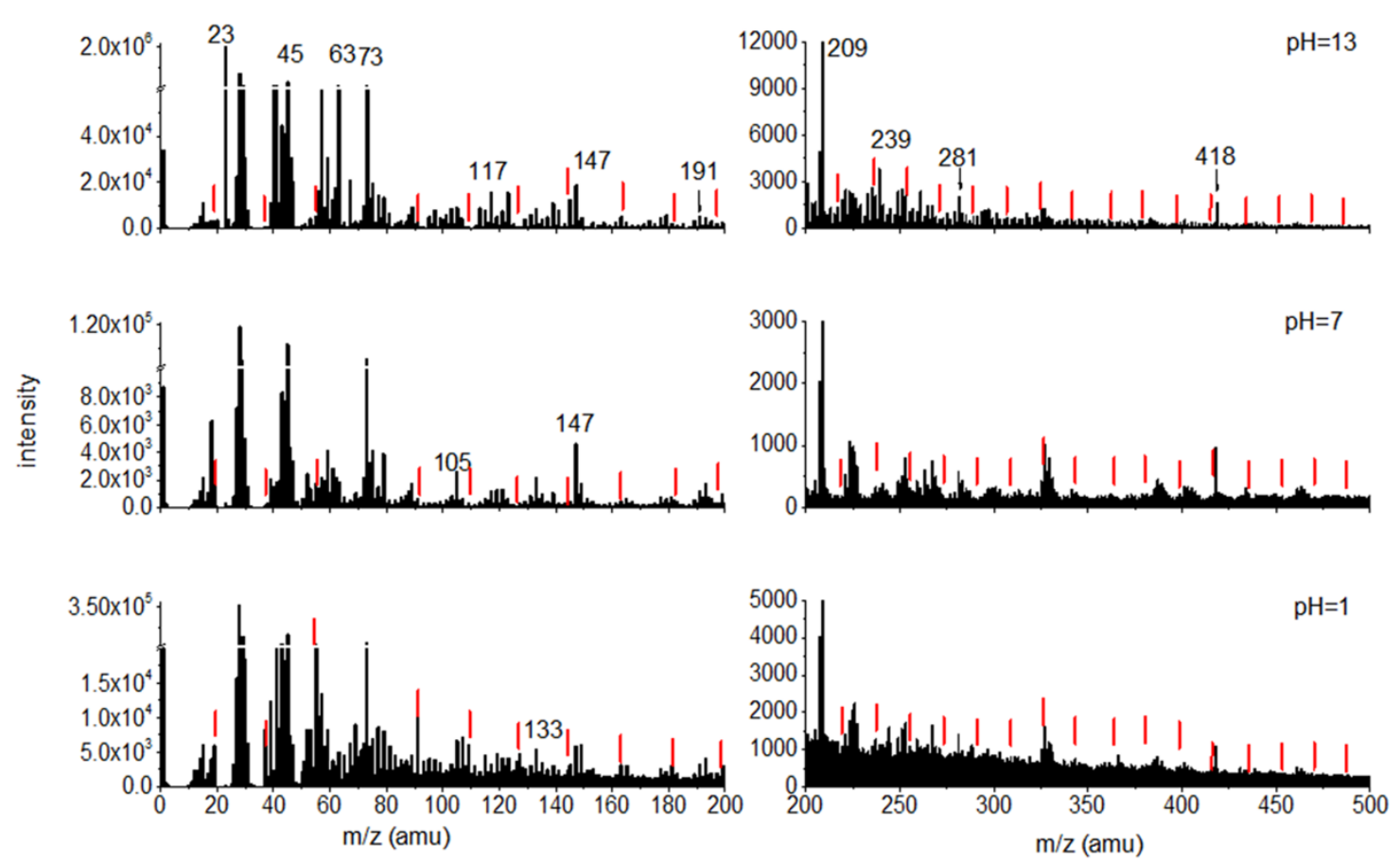

Figure 2. SIMS spectral results of synthesized boehmite particles under various $\mathrm{pH}$ conditions in the positive ion mode: (a) $\mathrm{pH} \mathrm{1,} \mathrm{(b)} \mathrm{pH} \mathrm{7,} \mathrm{and} \mathrm{(c)} \mathrm{pH} 13$. Red bars indicate locations of water clusters. 